\title{
ON THE COMPARISON OF EVOLUTION GALERKIN AND DISCONTINUOUS GALERKIN SCHEMES
}

\author{
K. BAUMBACH and M. LUKÁČOVÁ-MEDVIĎOVÁ \\ Institute of Numerical Simulation, Hamburg University of Technology, \\ 21079 Hamburg, Germany, \\ Emails: katjabaumbach@gmx.de, lukacova@tu-harburg.de \\ www.tu-harburg.de/math/hp/lukacova
}

\begin{abstract}
The aim of this paper is to compare some recent numerical schemes for solving hyperbolic conservation laws. We consider the flux vector splitting finite volume methods, finite volume evolution Galerkin scheme as well as the discontinuous Galerkin scheme. All schemes are constructed using time explicit discretization. We present results of numerical experiments for the shallow water equations for continuous as well as discontinuous solutions and compare accuracy and computational efficiency of the considered methods.
\end{abstract}

Keywords: shallow water equations, discontinuous Galerkin methods, evolution Galerkin methods, hyperbolic conservation laws

\section{Introduction}

Hyperbolic conservation laws can be found in many practical problems appearing in physics, biology or engineering. Among many applications which can be modelled by them, phenomena in fluid dynamics, elastodynamics, biomechanics, astrophysics, geophysics and traffic systems can be mentioned. Although theoretical study of these complex systems is not yet finished, we do not have a complete existence and uniqueness theory for multidimensional systems of hyperbolic conservation laws, for example, there is an extensive literature on numerical schemes for solutions of hyperbolic conservation laws, see, e.g., Feistauer et al. ${ }^{8}$, Cockburn et al. ${ }^{5}$, Kröner ${ }^{12}$, LeVeque $^{13}$, Toro $^{25}$ and many others.

The goal of this paper is to present results of a comparative study of some recent numerical schemes, which are commonly used in order to approximate hyperbolic conservation laws. In particular, we have chosen the classical finite volume dimensional splitting scheme, finite volume evolution Galerkin 
scheme, which belongs to the class of genuinely multidimensional schemes as well as the discontinuous Galerkin method. Except Dedner et al. ${ }^{6}$ we have found no other systematic studies in the literature on the comparison between the finite volume-type schemes and the discontinuous Galerkin schemes for multidimensional hyperbolic conservation laws.

The paper is organized as follows. We give brief descriptions of the methods in Sections 1.-3. In Section 4 we present some numerical experiments for two-dimensional shallow water equations. For further comparisons for scalar nonlinear hyperbolic equations see also Baumbach ${ }^{1}$.

Let us consider a two-dimensional system of hyperbolic conservation laws

$$
\partial_{t} \boldsymbol{u}+\partial_{x_{1}} \boldsymbol{f}_{1}(\boldsymbol{u})+\partial_{x_{2}} \boldsymbol{f}_{2}(\boldsymbol{u})=0, \quad \boldsymbol{x}=\left(x_{1}, x_{2}\right)^{T} \in \Omega,
$$

where $\boldsymbol{f}_{k}=\boldsymbol{f}_{k}(\boldsymbol{u}), k=1,2$, represent given physical flux functions and the conservative variables are $\boldsymbol{u}=\left(u_{1}, \ldots, u_{m}\right)^{T} \in \mathbb{R}^{m}$.

Divide a computational domain $\Omega$ into a finite number of regular finite volumes $\Omega_{i j}=\left[x_{i}-\Delta x_{1} / 2, x_{i}+\Delta x_{1} / 2\right] \times\left[y_{j}-\Delta x_{2} / 2, y_{j}+\Delta x_{2} / 2\right], i, j \in \mathbb{Z}$, $\Delta x_{1}, \Delta x_{2}$ are mesh steps. Further, we denote by $\boldsymbol{U}_{i j}^{n}$ the piecewise constant approximate solution on a mesh cell $\Omega_{i j}$ at time $t_{n}$ and start with initial approximations obtained by the integral averages $\boldsymbol{U}_{i j}^{0}=\int_{\Omega_{i j}} \boldsymbol{U}(\cdot, 0)$.

The finite volume method (FVM) can be formulated as follows

$$
\boldsymbol{U}^{n+1}=\boldsymbol{U}^{n}-\frac{\Delta t}{\Delta x_{k}} \sum_{k=1}^{2} \delta_{x_{k}} \boldsymbol{g}_{k},
$$

where $\Delta t$ is a time step, $\delta_{x_{k}}$ stays for the central difference operator in the $x_{k}$-direction, $k=1,2$, and $\boldsymbol{g}_{k}$ represents an approximation to the edge flux, the so-called numerical flux.

A large class of finite volume schemes can be found in literature. They are constructed using different approximations of flux functions, e.g. fluxvector splitting methods of Vijayasundaram, Van Leer, Steger-Warming, Roe, as well as central schemes of Lax-Friedrichs, Lax-Wendroff, Tadmor, Kurganov, and many others, see, e.g., Ref. 8 and the references therein.

In this paper we work with the numerical flux of Van Leer, which is given in the following way. Let $\mathbb{A}_{k}$ be the Jacobian matrix of the flux function $\boldsymbol{f}_{k}$ having real eigenvalues $\lambda_{k, 1}, \ldots, \lambda_{k, m}$. Further, let $\left|\mathbb{A}_{k}\right|$ be the matrix having eigenvalues $\left|\lambda_{k, 1}\right|, \ldots,\left|\lambda_{k, m}\right|, k=1,2$. Now, the numerical flux of Van Leer reads

$$
\boldsymbol{g}_{k}^{V L}:=\boldsymbol{g}_{k}^{V L}(\boldsymbol{u}, \boldsymbol{v})=\frac{1}{2}\left\{\boldsymbol{f}_{k}(\boldsymbol{u})+\boldsymbol{f}_{k}(\boldsymbol{v})-\left|\mathbb{A}_{k}\left(\frac{\boldsymbol{u}+\boldsymbol{v}}{2}\right)\right|\right\}
$$


with $\boldsymbol{u}, \boldsymbol{v}$ being the neighbouring values of the approximate solution to the cell interface; e.g., we have for the first order scheme $\boldsymbol{u}=\boldsymbol{U}_{i j}^{n}$ and $\boldsymbol{v}=\boldsymbol{U}_{i+1, j}^{n}$ for the right vertical edge of the mesh cell $\Omega_{i j}$. To obtain second order schemes suitable bilinear recovery $R_{h}$ has to be used. Higher order FVM typically uses the Runge-Kutta time approximation, cf. Section 3. For simplicity we present here results using only a regular rectangular mesh. The generalization of the FVM on irregular meshes is standard, see, e.g., Refs. 8,12 , for further details.

\section{Finite volume evolution Galerkin methods}

The evolution (or characteristic) Galerkin schemes have been firstly introduced by Bill Morton, Endre Süli and their collaborators for scalar problems and one-dimensional systems; see, e.g., Refs. 14, 15. This research was motivated by the pioneering work of Butler ${ }^{2}$ and related works of $\operatorname{Prasad}^{22,23}$. In 1997 Stella Ostkamp ${ }^{21}$ generalized these schemes to approximate the solution of the wave equation system as well as the Euler equations of gas dynamics in two space dimensions. In 2000 Lukáčová, Morton and Warnecke ${ }^{16}$ derived first new EG-schemes with better accuracy and stability. In the recent works of Lukáčová et al. ${ }^{17-19,11,20}$ a genuinely multidimensional finite volume evolution Galerkin (FVEG) method has been developed, studied extensively from theoretical as well as experimental point of view and applied to various applications. The method is based on the theory of bicharacteristics, which is combined with the finite volume framework. It can be also viewed as a predictor-corrector scheme; in the predictor step data are evolved along the bichracteristics, or along the bicharacteristic cone, in order to determine approximate solution on cell interfaces. In the corrector step the finite volume update (2) is done. Thus, we do not use here any one-dimensional approximate Riemann solver as it is done in the classical FVM, cf. (3). Instead the intermediate solution on cell interfaces is computed by means of an approximate evolution operator.

The cell interface fluxes are approximated using an approximate evolution operator denoted by $E_{\Delta t / 2}$ and averaged along the cell interface denoted by $\mathcal{E}$. The approximate evolution operator $E_{\Delta t / 2}$ evolves approximately solution up to the intermediate time step $t_{n}+\Delta t / 2$, i.e.

$$
\boldsymbol{g}_{k}^{E G}:=\frac{1}{|\mathcal{E}|} \int_{\mathcal{E}} \boldsymbol{f}_{k}\left(E_{\Delta t / 2} \boldsymbol{U}^{n}\right) \mathrm{d} S .
$$

The key ingredient of the finite volume evolution Galerkin scheme is the approximate evolution operator $E_{\Delta t / 2}$, which takes into account all of the 
infinitely many directions of wave propagations. It can be constructed for any multidimensional system of hyperbolic conservation laws using the theory of bicharacteristics. We refer the reader to Refs. 18, 19, 20 for examples of such approximate evolution operators for the Euler equations, shallow water equations as well as for the wave equation system, cf. also Section 4. Note that in the case of one-dimensional systems as well as multidimensional scalar equations the FVEG method reduces to classical upwind and characteristic schemes, respectively. Generalization of the FVEG methods on irregular triangular meshes has been done by Qurrat-ul-Ain ${ }^{26}$.

\section{Discontinuous Galerkin methods}

The discontinuous Galerkin (DG) methods are recently very popular methods to solve partial differential equations. The higher order DG method has been applied to nonlinear hyperbolic conservation laws as early as in 1989 by Cockburn and Shu ${ }^{3}$. Afterwards the DG method enjoys a rapid development, see, e.g. Cockburn et al. ${ }^{5,4}$, Feistauer et al. ${ }^{7,8}$, Houston, Süli et al. ${ }^{9,10,24}$ and the references therein. The DG methods compromise the ideas of numerical fluxes and limiters into a framework of finite element methods. Like all finite element methods, the DG methods can handle complex geometries and incorporate naturally boundary conditions. Important practical advantage of the DG methods in comparison with the finite volume-type schemes is the fact, that the DG methods have more compact stencils than the FV methods.

Let us denote by $h$ the mesh step, i.e. $h=\max \left(\Delta x_{1}, \Delta x_{2}\right)$, and let $\boldsymbol{X}_{h}$ be a space of piecewise polynomials of degree $p$ on a regular rectangular mesh $\Omega_{h}, \Omega_{h}=\left\{\Omega_{i j}\right\}_{i, j \in \mathbb{Z}}$. Thus,

$$
\boldsymbol{X}_{h}=\left\{\boldsymbol{v}_{h} \in B V\left(\Omega_{h} ; \mathbb{R}^{m}\right) ;\left.\boldsymbol{v}_{h}\right|_{\Omega_{i j}} \in P^{p}\left(\Omega_{i j}\right) \text { for all } \Omega_{i j}\right\},
$$

where $B V$ denotes the space of functions with bounded variation and $P^{p}\left(\Omega_{i j}\right)$ is the set of all polynomials of degree $p$ on the mesh cell $\Omega_{i j}$. In this paper we are interested in comparisons of second order methods, which means that we set $p=1$ and work with piecewise bilinear approximations. Furthermore, the following standard notation is introduced: $\left.\left[\boldsymbol{v}_{h}\right]\right|_{\mathcal{E}}$ denotes the jump of the function $\boldsymbol{v}_{h}$ on the cell interface $\mathcal{E}$. For example, if $\mathcal{E}=\mathcal{E}_{i j, i^{\prime} j^{\prime}}$ is the cell interface between the mesh cell $\Omega_{i j}$ and its neighbour $\Omega_{i^{\prime} j^{\prime}}$, then $\left.\left[\boldsymbol{v}_{h}\right]\right|_{\mathcal{E}_{i j, i^{\prime} j^{\prime}}}:=\left(\left.\boldsymbol{v}_{i j}\right|_{\mathcal{E}}-\left.\boldsymbol{v}_{i^{\prime} j^{\prime}}\right|_{\mathcal{E}}\right) \boldsymbol{n}$, where $\boldsymbol{n}$ denotes the outer normal on $\mathcal{E}$. 
Derivation of the DG methods is based on the so-called variational formulation of the hyperbolic system. The semi-discrete DG approximation of (1) is given by the following initial value problem

$$
\begin{aligned}
& \boldsymbol{U}_{h} \in C^{1}\left([0, T] ; \boldsymbol{X}_{h}\right), \\
& \frac{d}{d t}\left(\boldsymbol{U}_{h}(t), \boldsymbol{\varphi}_{h}\right)-\left(\boldsymbol{f}\left(\boldsymbol{U}_{h}(t)\right), \nabla \boldsymbol{\varphi}_{h}\right)+\left(\boldsymbol{g}\left(\boldsymbol{U}_{h}(t)\right),\left[\boldsymbol{\varphi}_{h}\right]\right)_{\mathcal{E}}=0, \quad \forall \boldsymbol{\varphi}_{h} \in \boldsymbol{X}_{h}, \\
& \boldsymbol{U}_{h}(0)=\boldsymbol{U}_{h}^{0} .
\end{aligned}
$$

Here $(\cdot, \cdot)$ represents the $L^{2}$ scalar product, $(\cdot, \cdot)_{\mathcal{E}}$ denotes the $L^{2}$ scalar product on the set of all interfaces $\mathcal{E}, \boldsymbol{f}=\left(\boldsymbol{f}_{1}, \boldsymbol{f}_{2}\right)^{T}$, and $\boldsymbol{g}$ denotes the numerical flux, which approximates physical fluxes along the cell interface $\mathcal{E}$. Thus, we have

$$
\left.\boldsymbol{g}\left(\boldsymbol{U}_{h}(t)\right)\right|_{\mathcal{E}_{i j, i^{\prime} j^{\prime}}}:=\boldsymbol{g}\left(\left.\boldsymbol{U}_{i j}\right|_{\mathcal{E}},\left.\boldsymbol{U}_{i^{\prime} j^{\prime}}\right|_{\mathcal{E}}\right)
$$

The system (6) can be equivalently rewritten in the so-called matrix form using the standard piecewise bilinear basis of $\boldsymbol{X}_{h}$ having vertices as degrees of freedom. Since the approximation is discontinuous, the mass matrix is block diagonal and easily invertible. For the above choice of grid and basis functions the entries of the mass matrix, which are the integrals of products of bilinear basis functions, can be evaluated exactly.

We will now consider the integral terms in (6). If a second order convergence has to be obtained a suitable choice of quadrature rules is necessary. More precisely, in Ref. 4, Cockburn et al. state that for second order convergence on a rectangular grid, the approximations of the volume integrals have to be exact for polynomials of degree 4 and the approximations of the cell interface integrals have to be exact for polynomials of degree 5 . We will use the Gauss quadratures for the approximation of integral terms. Using $k$ points, the Gauss quadrature is exact for polynomials of degree $2 k-1$. Consequently, on a rectangular grid we have to use the Gauss quadrature with 3 points for the cell interface integrals and $3 \times 3$ points for the volume integrals.

Further, in order to obtain fully discrete DG method a suitable time discretization has to be applied. Since the above described numerical schemes, FVM and FVEG schemes are explicit in time, we consider here only time explicit DG schemes, too. Following works of Cockburn et al. ${ }^{5}$ we use the sec- 
ond order Runge-Kutta discretization in time, also called the Heun method, which is total variation diminishing.

Since the Runge-Kutta discretization is explicit in time a CFL stability condition has to be imposed to guarantee the stability of the scheme. According to Cockburn ${ }^{5}$ it is known that using polynomials of degree $p$ and a $p+1$-stage Runge-Kutta approximation of order $p+1$ the von Neumann analysis for a linear one-dimensional advection equation yields the following stability condition

$$
|a| \frac{\Delta t}{\Delta x} \leq \frac{1}{2 p+1}
$$

where $a$ stays for the advection speed. In our case, $p=1$ which implies the CFL stability limit 0.33 . This stability limit has been indeed confirmed by our numerical experiments, too, cf. Section 4.3.

When approximating discontinuous solutions with piecewise polynomials of higher order, local maxima and minima can arise in the numerical solution, which are not physical. To avoid this effect a limiter has to be applied at each time level. In the finite volume methods as well as in the finite volume evolution Galerkin scheme we have applied the so-called minmod limiter, cf. Ref. 13. In the DG methods a generalized minmod limiter, see Ref. 5, has been applied.

\section{Numerical experiments}

In this section we illustrate behaviour of the methods described above on several examples for the system of shallow water equations. We present results for the discontinuous solution of circular dam break problem and compare the accuracy and computational efficiency of the second order FVM, FVEG and DG methods for a smooth solution. Further results of extensive experimental study can be found in Baumbach ${ }^{1}$.

\subsection{Shallow Water Equations}

The shallow water equations play an important role in modelling of a variety of free surface flows, such as oceanographic, atmospheric and geophysical flows, flows in lakes and rivers. Such flows are all characterized by negligible vertical scales in comparison to horizontal scales.

The homogeneous shallow water equations written in conservative variables have the following form

$$
\partial_{t} \boldsymbol{U}+\partial_{x_{1}} \boldsymbol{f}_{1}(\boldsymbol{U})+\partial_{x_{2}} \boldsymbol{f}_{2}(\boldsymbol{U})=0
$$


with

$$
\boldsymbol{U}=\left(\begin{array}{c}
h \\
h u \\
h v
\end{array}\right), \quad \boldsymbol{f}_{1}=\left(\begin{array}{c}
h u \\
h u^{2}+\frac{1}{2} g h^{2} \\
h u v
\end{array}\right), \quad \boldsymbol{f}_{2}=\left(\begin{array}{c}
h v \\
h v u \\
h v^{2}+\frac{1}{2} g h^{2}
\end{array}\right) .
$$

Here $h$ denotes the water depth, $u, v$ are vertically averaged velocity components and $g$ stays for the gravitational acceleration, we set $g=10$ in our computations.

As described in Section 2 the predictor step in the FVEG scheme is based on the approximate evolution operator. In order to keep the paper selfcontained we present here the approximate evolution operator for the shallow water equations (9), (10).

The predicted solution at cell interfaces is obtained by a suitable combination of two operators $E_{\Delta}^{\text {const }}$ and $E_{\Delta}^{\text {bilin }}$. We use $E_{\Delta}^{\text {bilin }}$ to evolve slopes and $E_{\Delta}^{\text {const }}$ to evolve the corresponding constant part in order to preserve conservativity, i.e.

$$
E_{\Delta t / 2} \boldsymbol{U}^{n}:=E_{\Delta t / 2}^{\text {bilin }} R_{h} \boldsymbol{U}^{n}+E_{\Delta t / 2}^{\text {const }}\left(1-\mu_{x}^{2} \mu_{y}^{2}\right) \boldsymbol{U}^{n},
$$

where $\mu_{x}^{2} U_{i j}=1 / 4\left(U_{i+1, j}+2 U_{i j}+U_{i-1, j}\right)$; an analogous notation is used for the $y$-direction. We have denoted by $R_{h}$ a suitable bilinear recovery. Note that $R_{h} \boldsymbol{U}$ is a continuous bilinear function if no limiters are used. It has been shown in Ref. 19 that the combination (11) yields the best results with respect to accuracy as well as stability among other possible second order FVEG schemes. Actually, the CFL stability limit of the FVEG is close to a natural stability limit of 1 , cf. Ref. 19. Using the theory of bicharacteristics the following approximate evolution operators for the shallow water equations have been derived, cf. Refs. 17, 19 .

For constant approximate functions the approximate evolution operator $E_{\Delta}^{\text {const }}$ reads

$$
\begin{gathered}
h(P)=\frac{1}{2 \pi} \int_{0}^{2 \pi}\left[h(Q)-\frac{\tilde{c}}{g} u(Q) \operatorname{sgn}(\cos \theta)-\frac{\tilde{c}}{g} v(Q) \operatorname{sgn}(\sin \theta)\right] \mathrm{d} \theta, \\
\begin{array}{c}
u(P)=\frac{1}{2 \pi} \int_{0}^{2 \pi}\left[-\frac{g}{\tilde{c}}\left(h(Q) \operatorname{sgn}(\cos \theta)+u(Q)\left(\cos ^{2} \theta+\frac{1}{2}\right)\right.\right. \\
+v(Q) \sin \theta \cos \theta] d \theta .
\end{array}
\end{gathered}
$$


If the piecewise bilinear functions are used the approximate evolution operator $E_{\Delta}^{b i l i n}$ is given as follows

$$
\begin{aligned}
h(P)= & h\left(Q_{0}\right)+\frac{1}{4} \int_{0}^{2 \pi}\left(h(Q)-h\left(Q_{0}\right)\right) \mathrm{d} \theta-\frac{1}{\pi} \int_{0}^{2 \pi}\left[\frac{\tilde{c}}{g} u(Q) \cos \theta+\frac{\tilde{c}}{g} v(Q) \sin \theta\right] \mathrm{d} \theta, \\
u(P)= & u\left(Q_{0}\right)-\frac{1}{\pi} \int_{0}^{2 \pi} \frac{g}{\tilde{c}} h(Q) \cos \theta \mathrm{d} \theta \\
& +\frac{1}{4} \int_{0}^{2 \pi}\left[3 u(Q) \cos ^{2} \theta+3 v(Q) \sin \theta \cos \theta-u(Q)-\frac{1}{2} u\left(Q_{0}\right)\right] \mathrm{d} \theta .
\end{aligned}
$$

The analogous equations hold for the second velocity component $v$. Here $P=\left(x, y, t_{n}+\Delta t / 2\right)$ is the apex of the so-called bicharacteristic cone, $Q_{0}=\left(x-\tilde{u} \Delta t, y-\tilde{v} \Delta t, t_{n}\right)$ denotes the center of the sonic circle, $Q=$ $\left(x-\tilde{u} \Delta t / 2+\tilde{c} \Delta t / 2 \cos \theta, y-\tilde{v} \Delta t / 2+\tilde{c} \Delta t / 2 \sin \theta, t_{n}\right)$ denotes a point at the perimeter of the sonic circle at time $t_{n}$. The local velocities, which have been obtained by a suitable linearization, are denoted by $\tilde{u}, \tilde{v}, \tilde{c}=\sqrt{g \tilde{h}}$. We refer a reader to Refs. 17, 18, 19 for more details on the derivation of approximate evolution operators.

\subsection{Dam break problem}

Consider the dam break problem with the following initial data

$$
\begin{aligned}
& h=0.1, u=0, v=0, \quad\|\boldsymbol{x}\| \geq 0.3, \\
& h=1, \quad u=0, v=0, \quad\|\boldsymbol{x}\|<0.3 .
\end{aligned}
$$

The computational domain $[-1,1] \times[-1,1]$ was divided into $100 \times 100$ mesh cells. We set the CFL number to 0.45 and compute the solution until $T=$ 0.25. Absorbing boundary conditions are implemented by extrapolation of all solution components. We have used the Van Leer numerical flux for the FVM as well as for the DG method, cf. (3),(7). In Ref. 1 other classical one-dimensional numerical fluxes have been tested for the DG method, too. Their influence on the resulting numerical solutions was only marginal.

Cell interface integrals (4) in the FVEG method have been approximated by the Simpson rule, whereas we have used, according to Cockburn et al. ${ }^{5}$, the 3-point Gauss quadrature rule for the DG method. In the second order FVM a midpoint rule is used in order to approximate cell interface flux integrals.

Figure 1 shows a 3D graph of the water surface $h$ for the FVM, other methods yield analogous 3D graphs. Graphs of the water surface $h$ along 
the diagonal direction $x=y$ are presented in Figure 2. Although the global solution behaviour is similar, some differences can be noticed in the shock resolution, see Figures 3 and 4 for zoom plots of the water surface for $x=y$ and $y=0$ slices. The FVEG method yields the sharpest shock resolution for both directions. Interestingly, for the diagonal direction it is, in fact, comparable with the shock resolution of the FVM. The DG method is slightly more dissipative on the shock. Note that the shock resolution is influenced by the used limiters, too, see also Ref. 6 for other choices of limiters.

\subsection{Accuracy and efficiency tests}

The aim of this experiment is to compare the accuracy and efficiency of the second order FV, FVEG and DG methods. We consider the initial value problem for the shallow water equations with smooth initial data

$$
\begin{aligned}
& h=0.25, \\
& u=1+0.5 \sin (\pi y)+0.25 \cos (\pi x), \\
& v=1+0.25 \sin (\pi x)+0.5 \cos (\pi y), \quad[x, y] \in[-1,1] \times[-1,1] .
\end{aligned}
$$

The computational domain $[-1,1] \times[-1,1]$ was consecutively divided into $20,40, \ldots, 320$ mesh cells in each direction. We have implemented periodic boundary conditions and computed the solution until $T=0.2$. Note, that due to the smooth solution no limiter has been used. In order to compare accuracy of all methods we first set $\mathrm{CFL}=0.2$, since the $\mathrm{DG}$ method starts to be unstable from $\mathrm{CFL}=0.3$. This is in accordance with the stability analysis for linear scalar equation, cf. (8) for $p=1$. Tables 1-3 show the accuracy study and the experimental order of convergence (EOC) for the DG, FVEG, $\mathrm{FV}$ methods using $\mathrm{CFL}=0.2$, respectively. Since the exact solution is not known the global relative error is used in order to evaluate the experimental order of convergence. This is computed in the following way using three meshes of sizes $N_{1}, N_{2}:=N_{1} / 2, N_{3}:=N_{2} / 2$, respectively

$$
\mathrm{EOC}=\log _{2} \frac{\left\|U_{N_{2}}^{n}-U_{N_{3}}^{n}\right\|}{\left\|U_{N_{1}}^{n}-U_{N_{2}}^{n}\right\|} .
$$

Here $U_{N}^{n}$ is the approximate solution on the mesh with $N \times N$ cells.

We can clearly see that all methods yield second order accuracy. The FVEG scheme yields the best accuracy among all three methods in both the $L^{1}$ as well as $L^{2}$ norms. In fact, it is twice as accurate as the FVM and the DG scheme. Further, the relative error for the DG scheme is slightly better than that of the FVM. Analogous results have been obtained for the DG 
and FV methods by Dedner et al. showing that for smooth solutions the DG methods are more accurate than the FVM.

Further, we compare the accuracy of the FVEGM and FVM for higher CFL numbers. In Tables 4 and 5 we present the EOC results for $\mathrm{CFL}=0.6$. No results are presented for the DG scheme, which is unstable for CFL > 0.3. The FVEG method is about 10 times more accurate than the standard FVM. Note that we have obtained analogous results of accuracy study in our recent paper on well-balanced schemes, see Ref. 20.

Table 6 gives the overview of the computational costs for all of the methods, the DG, FVEG as well as classical FV method. We clearly see that the DG method is the most expensive one, being about 4 times slower than the FV method and about 3 times slower than the FVEG method. The CPU time of the FVEG method is slightly worse than that of the classical FV methods. Of course, the cell interface flux integration requires three quadrature points for the DG and the FVEG methods, whereas there is only one quadrature point, the midpoint, in the classical second order FVM.

Figure 5 illustrates the CPU/accuracy behaviour graphically. We use the logarithmic scale on $x-, y-$ axis. On the $y$ - axis the $L^{1}$ relative error for the vector of conservative variables is depicted. Figure 5 indicates that for smooth solutions the FVEG scheme is the most efficient in the class of compared second order schemes. We should point out that no attempt has been made in order to optimize the codes with respect to their CPU performance.

\section{Conclusion}

In this paper we have compared three recent second order methods for hyperbolic conservation laws, namely the classical flux-vector splitting finite volume schemes, the finite volume evolution Galerkin schemes and the discontinuous Galerkin schemes. For smooth solution the finite volume evolution Galerkin scheme yields the best accuracy and computational efficiency. Further, we have shown that all considered schemes approximate discontinuous solutions in a correct way; the FV-type schemes yield sligthly sharper shock resolution. The present study has been done only on regular rectangular meshes. In future it will be interesting to extend this study for irregular triangular meshes and (time implicit) higher order schemes (e.g. third or fourth order), where the higher order discontinuous Galerkin methods can demonstrate their flexibility. 


\section{Acknowledgement}

This research has been supported partially by the Deutsche Forschungsgemeinschaft under the grant LU 11942/2-1. The authors gratefully acknowledge this support. Further, we would like to thank Marcus Kraft, Hamburg University of Technology, for providing numerical results of the FVEG method for the comparison test 4.3 .

\section{References}

1. Baumbach K. On the Comparison of the Finite Volume and Discontinuous Galerkin Methods. Diploma Thesis, Hamburg University of Technology, 2006.

2. Butler DS. The numerical solution of hyperbolic systems of partial differential equations in three independent variables. Proc. Roy. Soc. 1960; 225A:233-252.

3. Cockburn B, Shu CW. TVB Runge-Kutta local projection discontinuous Galerkin finite element method for conservation laws II: General framework. Math. Comput. 1989; 52:411-435.

4. Cockburn B, Shu CW. Runge-Kutta discontinuous Galerkin methods for convection-dominated problems. J. Sci. Comput. 2001; 16(3):173-261.

5. Cockburn B, Karniadakis GE, Shu CW. (Eds.) Discontinuous Galerkin Methods. Springer, Berlin, 2000.

6. Dedner A, Kröner D, Rohde C, Schnitzer T., Wesenberg M. Comparision of finite volume and discontinuous Galerkin methods of higher order for systems of conservation laws in multiple space dimensions. Geometric analysis and nonlinear partial differential equations Eds.: Hildebrandt, S. et al., Springer, Berlin, 573-589, 2003.

7. Dolejší V, Feistauer M, Schwab C. On some aspects of the discontinuous Galerkin finite element method for conservation laws. Math. Comput. Simul. 2003; 61: 333-346.

8. Feistauer M, Felcman J, Straškraba J. Mathematical and Computational Methods for Compressible Flow. Clarendon Press Oxford, 2003.

9. Houston P, Schwab Ch, Süli E. Discontinuous hp-finite element methods for advection-diffusion-reaction problems. SIAM J. of Num. Anal. 2002; 39(6):2133-2163.

10. Houston P, Jensen M, Süli E. hp-Version Discontinuous Galerkin Finite Element Methods with least-squares stabilization. J. of Scientific Comput. 2002 17(1):3-25.

11. Kröger T, Lukáčová-Medvid’ová M. An evolution Galerkin scheme for the shallow water magnetohydro-dynamic (SMHD) equations in two space dimensions. J. Comp. Phys. 2005; 206:122-149.

12. Kröner D. Numerical Schemes for Conservation Laws., Wiley and Teubner, 1996.

13. LeVeque RJ. Finite Volume Methods for Hyperbolic Problems. Cambridge University Press, Cambridge, 2002. 
14. Lin P, Morton KW, Süli E. Euler characteristic Galerkin scheme with recovery. $M^{2} A N$ 1993; 27(7):863-894.

15. Lin P, Morton KW, Süli E. Characteristic Galerkin schemes for scalar conservation laws in two and three space dimensions. SIAM J. Numer. Anal. 1997; 34(2):779-796.

16. Lukáčová-Medvid’ová M, Morton KW, Warnecke G. Evolution Galerkin methods for hyperbolic systems in two space dimensions. Math. Comp. 2000; 69:1355-1384.

17. Lukáčová-Medvid’ová M. Multidimensional bicharacteristics finite volume methods for the shallow water equations. In: Finite Volumes for Complex Applications, eds. R. Hérbin and D. Kröner, Hermes, 2002, 389-397.

18. Lukáčová-Medvid’ová M, Saibertová J, Warnecke G. Finite volume evolution Galerkin methods for nonlinear hyperbolic systems. J. Comp. Phys. 2002; 183:533-562.

19. Lukáčová-Medvid’ová M, Morton KW, Warnecke G. Finite volume evolution Galerkin (FVEG) methods for hyperbolic problems. SIAM J. Sci. Comput. 2004; 26(1):1-30.

20. Lukáčová-Medvidová M, Noelle S, Kraft M. Well-balanced finite volume evolution Galerkin methods for the shallow water equations. accepted to J. Comp. Phys., 2006.

21. Ostkamp S. Multidimensional characteristic Galerkin schemes and evolution operators for hyperbolic systems. Math. Meth. Appl. Sci. 1997; 20:11111125.

22. Prasad P, Ravindran R. Canonical form of a quasilinear hyperbolic system of first order equations. J. Math. Phys. Sci. 1984; 18(4):361-364.

23. Reddy AS, Tikekar VG, Prasad P. Numerical solution of hyperbolic equations by method of bicharacteristics. J. Math. Phys. Sci. 1982; 16(6):575603.

24. Süli E, Houston P, Senior B. hp-Discontinuous Galerkin finite element methods for hyperbolic problems: error analysis and adaptivity. International Journal for Numerical Methods in Fluids. Volume 2002; 40(1-2):153-169.

25. Toro EF. Riemann Solvers and Numerical Methods for Fluid Dyanmics, (2nd Edition), Springer Verlag, Berlin, Heidelberg, 1999.

26. Qurrat-ul-Ain. Multidimensional Schemes for Hyperbolic Conservation Laws on Triangular Meshes. Dissertation, University of Magdeburg, 2005. 


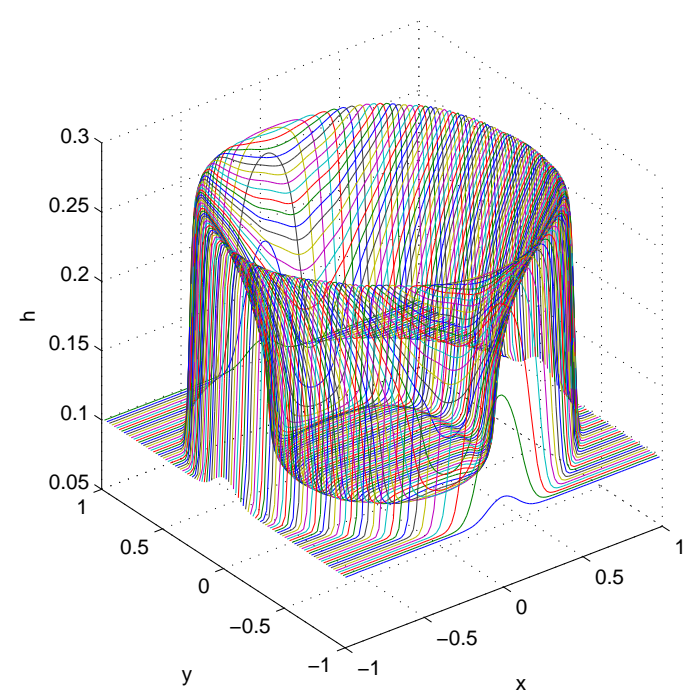

Fig. 1. 3D graph of the water surface obtained by the FVM using $100 \times 100$ cells.

Table 1. Convergence of the DG scheme, $C F L=0.2$.

\begin{tabular}{|c|c|c|c||c|}
\hline$N \times N$ cells & $\left\|U_{N / 2}^{n}-U_{N}^{n}\right\|_{L^{2}}$ & $E O C_{L^{2}}$ & $\left\|U_{N / 2}^{n}-U_{N}^{n}\right\|_{L^{1}}$ & $E O C_{L^{1}}$ \\
\hline \hline $40 \times 40$ & 0.003399 & & 0.008227 & \\
\hline $80 \times 80$ & 0.000841 & 2.014933 & 0.002039 & 2.012505 \\
\hline $160 \times 160$ & 0.000208 & 2.015522 & 0.000506 & 2.010652 \\
\hline $320 \times 320$ & 0.000052 & 2.000000 & 0.000126 & 2.005714 \\
\hline
\end{tabular}

Table 2. Convergence of the FVEG scheme, $C F L=0.2$.

\begin{tabular}{|c|c|c|c||c|}
\hline$N \times N$ cells & $\left\|U_{N / 2}^{n}-U_{N}^{n}\right\|_{L^{2}}$ & $E O C_{L^{2}}$ & $\left\|U_{N / 2}^{n}-U_{N}^{n}\right\|_{L^{1}}$ & $E O C_{L^{1}}$ \\
\hline \hline $40 \times 40$ & 0.001921 & & 0.004640 & \\
\hline $80 \times 80$ & 0.000445 & 2.109484 & 0.001066 & 2.121560 \\
\hline $160 \times 160$ & 0.000110 & 2.021743 & 0.000261 & 2.032406 \\
\hline $320 \times 320$ & 0.000028 & 1.990744 & 0.000065 & 1.999932 \\
\hline
\end{tabular}

Table 3. Convergence of the FV scheme, $C F L=0.2$.

\begin{tabular}{|c|c|c|c||c|}
\hline$N \times N$ cells & $\left\|U_{N / 2}^{n}-U_{N}^{n}\right\|_{L^{2}}$ & $E O C_{L^{2}}$ & $\left\|U_{N / 2}^{n}-U_{N}^{n}\right\|_{L^{1}}$ & $E O C_{L^{1}}$ \\
\hline \hline $40 \times 40$ & 0.004143 & & 0.009473 & \\
\hline $80 \times 80$ & 0.001009 & 2.037750 & 0.002313 & 2.034056 \\
\hline $160 \times 160$ & 0.000258 & 1.967483 & 0.000595 & 1.958804 \\
\hline $320 \times 320$ & 0.000066 & 1.966833 & 0.000152 & 1.968818 \\
\hline
\end{tabular}


14

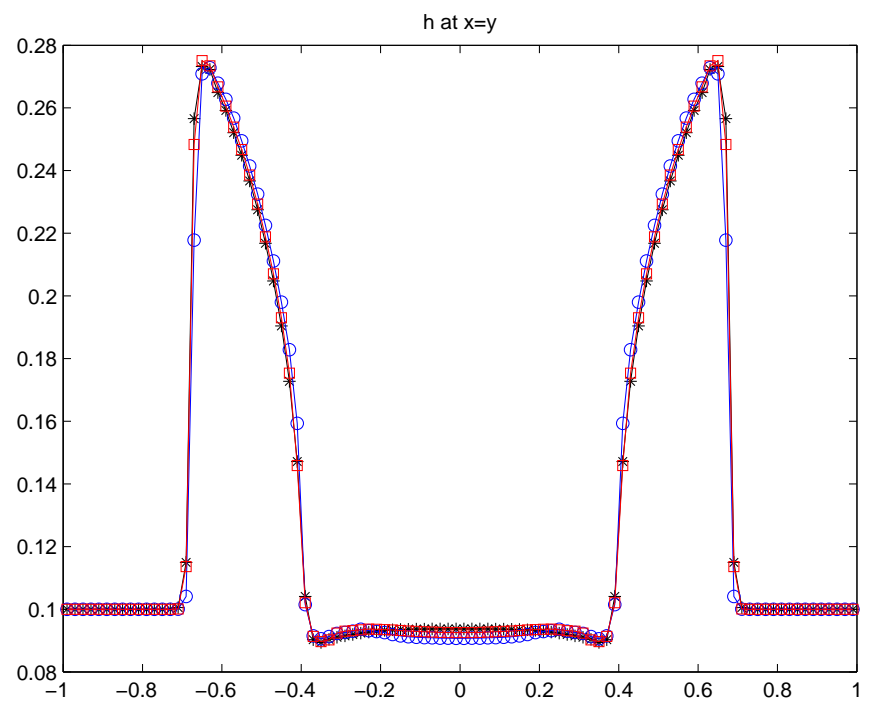

Fig. 2. Graphs of the water surface $h$ for $x=y$ using $100 \times 100$ cells; FVEG scheme (stars), the FVM (boxes) and the DG method (circles).

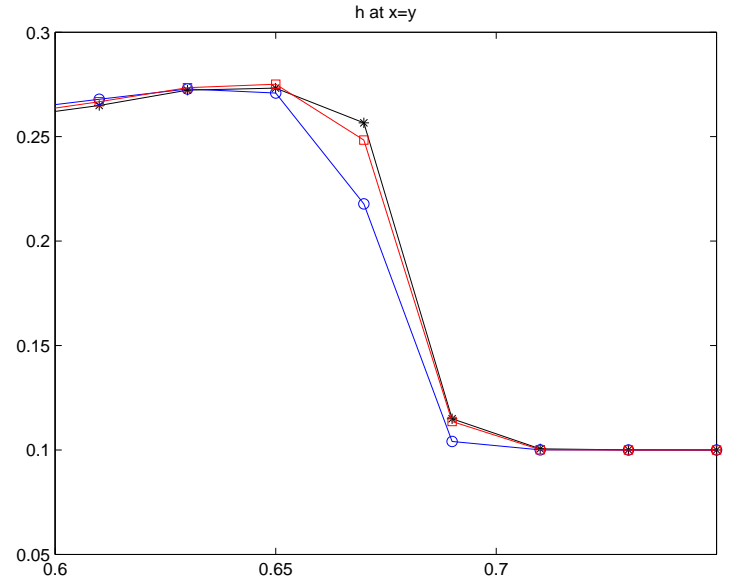

Fig. 3. Zoom of the shock resolution in $h$ for $x=y$ using $100 \times 100$ cells; FVEG scheme (stars), the FVM (boxes) and the DG method (circles). 


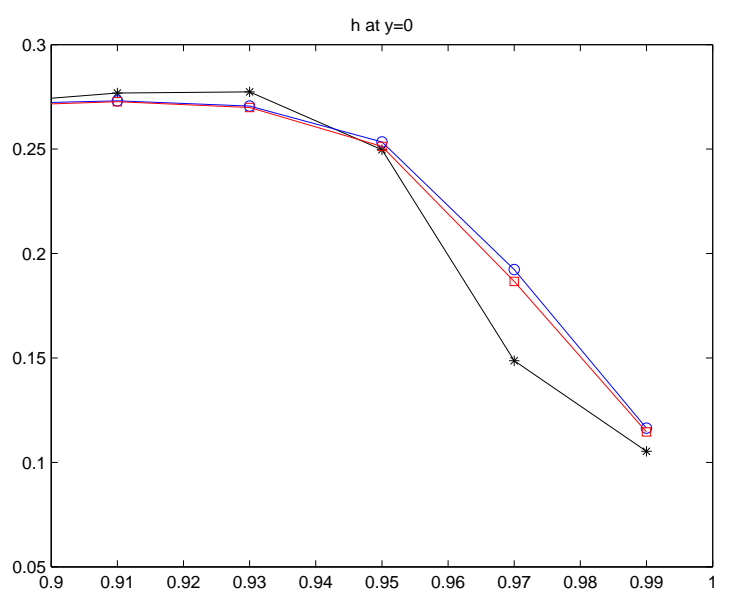

Fig. 4. Zoom of the shock resolution in $h$ for $y=0$ using $100 \times 100$ cells; FVEG scheme (stars), the FVM (boxes) and the DG method (circles).

Table 4. Convergence of the FVEG scheme, $C F L=0.6$.

\begin{tabular}{|c||c|c||c|c|}
\hline$N \times N$ cells & $\left\|U_{N / 2}^{n}-U_{N}^{n}\right\|_{L^{2}}$ & $E O C_{L^{2}}$ & $\left\|U_{N / 2}^{n}-U_{N}^{n}\right\|_{L^{1}}$ & $E O C_{L^{1}}$ \\
\hline \hline $40 \times 40$ & 0.000994 & & 0.002290 & \\
\hline $80 \times 80$ & 0.000174 & 2.516154 & 0.000405 & 2.500354 \\
\hline $160 \times 160$ & 0.000038 & 2.193481 & 0.000088 & 2.209174 \\
\hline $320 \times 320$ & 0.000009 & 2.021754 & 0.000021 & 2.044111 \\
\hline
\end{tabular}

Table 5. Convergence of the FV scheme, $C F L=0.6$.

\begin{tabular}{|c||c|c||c|c|}
\hline$N \times N$ cells & $\left\|U_{N / 2}^{n}-U_{N}^{n}\right\|_{L^{2}}$ & $E O C_{L^{2}}$ & $\left\|U_{N / 2}^{n}-U_{N}^{n}\right\|_{L^{1}}$ & $E O C_{L^{1}}$ \\
\hline \hline $40 \times 40$ & 0.004790 & & 0.010843 & \\
\hline $80 \times 80$ & 0.001268 & 1.917471 & 0.002851 & 1.927224 \\
\hline $160 \times 160$ & 0.000332 & 1.933300 & 0.000746 & 1.934221 \\
\hline $320 \times 320$ & 0.000085 & 1.965648 & 0.000192 & 1.958069 \\
\hline
\end{tabular}

Table 6. CPU time in seconds, $C F L=0.2$.

\begin{tabular}{|c|c|c|c|}
\hline$N \times N$ cells & DG scheme & FVEG scheme & FV scheme \\
\hline \hline $20 \times 20$ & 0.27 & 0.11 & 0.08 \\
\hline $40 \times 40$ & 2.14 & 0.85 & 0.53 \\
\hline $80 \times 80$ & 18.47 & 6.85 & 4.41 \\
\hline $160 \times 160$ & 151.57 & 53.01 & 37.2 \\
\hline $320 \times 320$ & 1216.84 & 437,10 & 296.38 \\
\hline $640 \times 640$ & 10660.43 & 3354.39 & 2560.55 \\
\hline
\end{tabular}




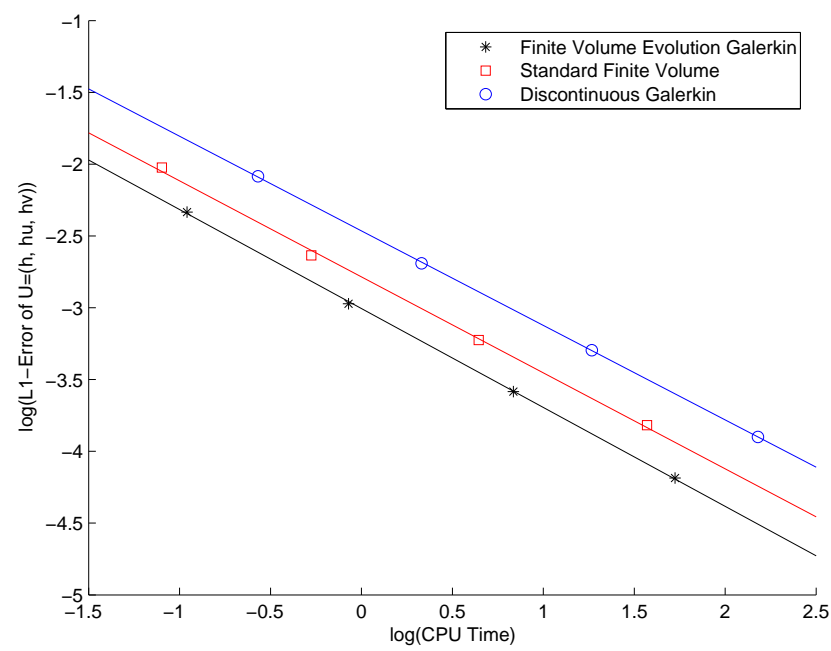

Fig. 5. Efficiency study: relative $L^{1}$ error over CPU-time for the FVEG scheme (stars), the FVM (boxes) and the DG method (circles). 